\title{
AMPLIANDO EL HORIZONTE DE LA AGROECOLOGÍA: PRINCIPIOS, PRÁCTICA Y CADENA DE VALOR
}

\author{
Eduardo Moyano Estrada ${ }^{a^{*}}$, Fernando E. Garrido Fernández ${ }^{a}$
}

\author{
a Instituto de Estudios Sociales Avanzados, IESA-CSIC (Córdoba, emoyano@iesa.csic.es; \\ fgarrido@iesa.csic.es
}

\section{Resumen}

La Agroecología es una forma integral de conocimiento enraizado en el saber ancestral del campesinado, en contraposición al más especializado de la agricultura moderna. El enfoque de la Agroecología analiza el funcionamiento del mundo agrícola contemplando globalmente las interacciones entre sus componentes (suelo, agua, plantas, animales...) y de éstos con los seres humanos. Los principios considerados esenciales de la Agroecología inspiran el desarrollo de prácticas agrarias concretas, destacando entre ellas la agricultura ecológica, pero también la agricultura de conservación, la producción integrada, los huertos urbanos... Las prácticas agrícolas y ganaderas inspiradas en la Agroecología pueden dar lugar a cadenas de valor si interaccionan con otros eslabones, como el comercio, la distribución, la transformación y el consumo. Esas cadenas pueden ser cortas (producción-consumo), medias (producción-comercio-consumo) o largas (producción-industria-distribución-consumo), dándose dinámicas específicas de interacción entre productores y agentes de la cadena alimentaria.

En esta comunicación, se revisan los principios de la Agroecología, planteando su flexibilización e incluyendo como "agroecológicas" prácticas agrícolas que, sin ser las tradicionales del campesinado, contribuyen a la sostenibilidad ecológica, social y económica de las áreas rurales.

Palabras clave: agroecología, flexibilización, cadena de valor, sostenibilidad

\section{Introducción}

El término "agroecología" se ha popularizado, hasta el punto de que es frecuente escucharlo en los más variados ambientes y en círculos de opinión muy diversos (IPES-Food, 2016) Desde los agricultores que practican la producción ecológica, hasta los movimientos campesinos que cuestionan el legado de la "Revolución Verde", pasando por las plataformas de consumidores que reivindican una relación más directa con los productores, todos ellos enarbolan la bandera de la Agroecología como un medio para cambiar los modelos de producción y consumo que predominan en el sector de la alimentación. Pero también encontramos grupos de agricultores que se consideran en sintonía con los principios de la Agroecología (FAO, 2018) y que apuestan por modelos más sostenibles de agricultura sin rechazar frontalmente el modelo agroquímico, introduciendo prácticas y técnicas agrícolas y ganaderas muy eficientes en cuanto a la utilización de insumos y a la explotación de los recursos naturales.

Aunque su visibilidad ha sido más evidente en los últimos 20 años, la Agroecología no es un concepto reciente. Si bien los primeros usos del término podrían situarse a principios del pasado siglo XX, sus significados, definiciones o enfoques han ido evolucionando y adaptándose a distintas sensibilidades y preocupaciones (Wezel et al., 2020). En ese sentido, como suele ocurrir con los conceptos dinámicos que se extienden en campos semánticos, grupos sociales y círculos de opinión tan amplios y variados, es frecuente que se produzca alguna confusión terminológica (Gliessman, 2018; Gallardo-López et al., 2018)). Eso es lo que viene ocurriendo con el término "agroecología".

Por un lado, y desde el punto de vista de las prácticas agrarias, algunos círculos de opinión tienden a identificar la Agroecología exclusivamente con la agricultura ecológica, como si ambos términos tuvieran el mismo significado (Wezel et al., 2009). Y, por otro lado, una gran variedad de técnicas que no son de producción ecológica, pero que se reclaman herederas de la Agroecología por el simple hecho de utilizar de forma sostenible los recursos naturales y preservar el equilibrio de los ecosistemas (es lo que sucede por ejemplo con la agricultura campesina de autoconsumo, la permacultura o los programas de custodia y manejo holístico de los territorios).

Respecto al primer caso, la identificación entre "agroecología" y "agricultura ecológica" no ocurre sólo en el lenguaje coloquial, sino también en el seno de la comunidad académica y también en el mundo asociativo, como lo prueba el hecho de que la propia Sociedad Española de Agricultura Ecológica (SEAE) lo sea también de Agroecología. En este sentido, pareciera como si los movimientos asociativos en pro de la producción ecológica se hubiesen apropiado de tal modo de los principios de la Agroecología, que hayan acabado por desdibujar las diferencias entre ambos términos. No obstante, dada la gran variedad de modelos de producción ecológica (en explotaciones de tipo familiar o en grandes empresas agrícolas) y su diferente integración en la cadena alimentaria (desde los que se desarrollan en circuitos cortos a los que se canalizan 
a través de la gran distribución), identificar sin matices la Agroecología y la agricultura ecológica no deja de ser una forma de simplificar la relación entre ambos términos.

Respecto a la tendencia contraria, la de ampliar sin límite el horizonte de la Agroecología, observamos que se ha extendido a un amplio abanico de prácticas agrícolas (Gallardo-López, et al., 2018), como las citadas de la agricultura integrada, de precisión o de conservación, y también de prácticas sociales que trascienden incluso el medio rural, como las que se promueven desde las ciudades y que están amparadas por el Pacto de Milán (2015). Entre estas prácticas sociales cabe destacar los huertos urbanos en sus diversas expresiones (comunitarios, individuales, solidarios...), los mercados institucionales (para contribuir al abastecimiento de alimentos a centros escolares y a grupos sociales vulnerables) o los barrios productores (aprovechando con fines agrícolas los solares abandonados que proliferan en los grandes municipios). En todos estos casos, el riesgo es que se amplíe tanto el concepto de Agroecología que, al incluir una variedad tan amplia de prácticas agrícolas y sociales, acabe por vaciarse de contenido y se pierda la esencia de su significado.

Sea como fuere, es evidente que existe alguna confusión en el panorama actual de la Agroecología hasta el punto que se confunde o diluye su verdadero significado. El propósito de esta comunicación es contribuir al debate sobre qué se entiende o puede englobarse en el concepto de Agroecología, proponiendo una renovación y/o flexibilidad de sus principios que ayuden a su mayor difusión y adaptación a los desafíos actuales.

\section{Dos formas de interpretar la Agroecología}

Las diversas formas de interpretar la Agroecología pueden sintetizarse, a efectos analíticos y utilizando el enfoque metodológico weberiano de los tipos ideales, en las dos siguientes versiones: una, que podríamos calificar de "esencialista" (y militante), y otra, que podría ser calificada de "pragmático" (y tecnocrático) (Moyano, 2021). La versión "esencialista" vela por mantener intactas las esencias de los principios agroecológicos tachando de revisionistas a todos los que intentan desnaturalizarlos con la excusa de ampliar el horizonte de la Agroecología. Por el contrario, la versión "pragmática" apuesta por flexibilizar esos principios con el fin de adaptarlos a los cambios que se producen en la agricultura, calificando de fundamentalistas a aquéllos que cierran filas en torno a un modo rígido de interpretarlos.

\subsection{La versión "esencialista"}

Siguiendo a Altieri (1995), los principios fundamentales de la Agroecología están enraizados en el conocimiento ancestral del campesinado, que es un conocimiento holístico e integral de la agricultura. El enfoque de la Agroecología es, por tanto, un modo integral y omnicomprensivo (no parcial ni sectorial) de analizar el funcionamiento del mundo agrícola contemplando de forma global las interacciones entre sus distintos componentes (suelo, agua, plantas, nutrientes, animales...) y entre éstos y los seres humanos.

Sobre estas prácticas ancestrales del campesinado se fundamentan los principios canónicos de la Agroecología. De esos principios, hay cuatro que se consideran esenciales: i) diversidad ecológica; ii) rotación de cultivos; iii) combinación de la agricultura y la ganadería, y iv) investigación participativa.

Para la versión "esencialista", los principios de la Agroecología chocan frontalmente con los que han estado rigiendo los destinos de la agricultura desde hace más de 50 años, concretamente los asociados a la ya mencionada Revolución Verde (mejora genética, biotecnología, mecanización, intensificación productiva, utilización de insumos químicos...). Para esta versión de la Agroecología, los principios de la Revolución Verde han hecho que la agricultura vire hacia modelos de especialización cada vez más integrados en los mercados y más dependientes de la industria y las grandes multinacionales (IPES-Food, 2016; Nicholls et al., 2016).

Esos modelos agrarios de elevada especialización e intensificación productiva son el centro de la crítica de los partidarios de una versión esencialista de la Agroecología por dos razones. La primera razón es socioeconómica, al entender que esa lógica atrapa al pequeño agricultor en una cadena de dependencia respecto de la industria de insumos y la gran distribución, sometiéndolo, además, a un creciente proceso de endeudamiento con las entidades financieras. La segunda razón es ecológica, al considerar que la especialización e intensificación productiva acaban siendo perjudiciales para el medio ambiente, la preservación de los ecosistemas y la biodiversidad, además de ser una de las causas del cambio climático (Sinclair, 2019).

De este modo, la Agroecología en su versión esencialista deja, por tanto, de ser sólo un paradigma científico para adquirir un carácter ideológico-político y militante, al presentarse como el ariete listo para luchar contra el modelo de agricultura industrializada impuesto por las grandes transnacionales y abogar por una transformación profunda del actual paradigma alimentario (Calle y Álvarez, 2021; Sevilla-Guzmán, 2021). 


\subsection{La versión "pragmática"}

Hay, no obstante, otra versión de la Agroecología que podríamos denominarla "pragmática". Es una versión más técnica que política (tecnocrática diríamos), circunscrita al ámbito de los enfoques holísticos dirigidos a la preservación de los ecosistemas. Están de acuerdo en reformar en lo posible los modelos de producción agrícola asociados a la Revolución Verde, pero sin rechazar las nuevas tecnologías ni demonizar a las industrias de insumos.

Según esta versión, se aceptarían como agroecológicas todas aquellas prácticas que contribuyan a la biodiversidad y la sostenibilidad medioambiental, con independencia de que utilicen o no tecnologías procedentes del complejo agroindustrial y de que las explotaciones donde se apliquen sean gestionadas de forma empresarial o familiar. Modelos agrícolas como los basados en la agricultura de precisión, la producción integrada o la agricultura de conservación serían agroecológicos para esta versión pragmática siempre que contribuyan a la preservación de los ecosistemas mediante la diversificación y la extensificación productiva. Asimismo, la agricultura ecológica sería una práctica agrícola más, y no la más relevante, de las que se inspiran en la agroecología. Además, las prácticas de agricultura urbana impulsadas desde las ciudades (huertos, corredores verdes...) y amparadas por el ya citado Pacto de Milán (2015) se declaran agroecológicas, pudiendo ser también contempladas como tales desde esta versión más pragmática y menos esencialista de la agroecología.

Es esta versión la que impregna documentos tales como las dos estrategias del Pacto Verde Europeo y algunos de los programas de la PAC como el programa agro-ambiental y el de los "ecoesquemas", incorporado éste a su primer pilar para el periodo 2021-2027. Asimismo, es también esta versión la que encarnan los gobiernos que, como hemos señalado, se oponen en FAO a que la Agroecología sea considerada un enfoque fundamental para avanzar en la sostenibilidad de la agricultura y pretenden circunscribirla al ámbito del conocimiento técnico y las prácticas agrícolas eliminando de ella todo su discurso de cambio y transformación social.

\section{Renovando los principios de la Agroecología}

En un mundo agrícola tan cambiante como el actual, con la creciente incorporación de las nuevas tecnologías, el uso de nuevas fuentes de energía y el avance de la mejora genética, es necesario precisar mejor el contorno de la Agroecología. Sería conveniente acotar bien qué entendemos por Agroecología, revisando algunos de sus principios canónicos, al igual que se viene haciendo con los conceptos de agricultura familiar o cooperativismo.

Por ejemplo, habría que preguntarse qué es hoy el conocimiento agrícola local (Elzen et al., 2017). Es verdad que, en algunas áreas del planeta, ese conocimiento sigue enraizado en la cultura campesina ancestral, pero en otras es un conocimiento permeable y mezclado ya con hábitos y prácticas foráneas, procedentes de culturas influidas por las formas modernas y tecnificadas de hacer agricultura.

También debería revisarse el principio agroecológico de la diversidad, y debatir si es pertinente continuar planteando el tema de la diversificación y rotación de cultivos o el de la combinación agricultura-ganadería al nivel de la explotación o a una escala más amplia (por ejemplo, un área de cultivo o un área ganadera).

Asimismo, sería conveniente preguntarse por el significado de otro de los principios de la Agroecología, el de la investigación participativa, en un mundo como el actual en el que las redes sociales abren enormes vías para nuevas formas de participación de los agricultores (física o virtual) en los asuntos relacionados con la gestión de sus explotaciones. Además, cada vez más se produce la interacción de los productores y las industrias en la cadena alimentaria, y cada vez es mayor la influencia de los consumidores en los distintos eslabones de dicha cadena ejerciendo lo que algunos autores denomina sus derechos de "ciudadanía alimentaria" (Lozano y Gómez Benito, 2014). Por eso el principio agroecológico de la participación debe, al menos, replantearse a la luz de estos cambios.

Algo similar habría que señalar sobre los circuitos cortos y los mercados de proximidad, que suelen ser asimilados como prácticas agroecológicas y que hoy se desarrollan tanto mediante una relación física entre productores y consumidores, como a través de internet. Por último, sería conveniente determinar en qué medida y hasta dónde puede extenderse el horizonte de la Agroecología en lo que respecta a la gran variedad de prácticas agrícolas que, como hemos comentado anteriormente, se desarrollan en el medio urbano y cuyos promotores apelan a los principios agroecológicos a la hora de presentarlos al conjunto de la comunidad (Tornaghi y Dehaene, 2020).

En una conferencia pronunciada en 2020 por el que fuera DG de FAO, Graziano da Silva, se planteaba la necesidad de renovar el enfoque de la Agroecología para adaptarlo al nuevo escenario de la agricultura, así como de buscar sinergias entre las diversas formas de interpretar los principios de la Agroecología, de tal modo que puedan converger en perspectivas innovadoras capaces de dar respuesta a los grandes desafíos de la agricultura y la producción de alimentos (Anderson et al., 2020). 


\section{Conclusiones}

En esta comunicación se ha tratado de exponer la necesidad de abordar la renovación de los principios de la Agroecología, ya que si no se actualizan puede que su campo de acción se vea cada vez más restringido. Si no se aborda ese debate, la Agroecología acabaría reduciendo su influencia sólo en aquellos territorios donde aún persisten modelos tradicionales de agricultura campesina; o terminaría siendo reapropiada por aquellos movimientos más activos y militantes de la agricultura ecológica en esas otras áreas donde los modelos campesinos han dejado ya de existir.

Realmente, si el objetivo último de la Agroecología es asegurar el equilibrio de los ecosistemas, preservar la biodiversidad, mejorar el bienestar y aumentar la autonomía de los agricultores en todo el planeta, y no sólo en una parte del mismo, quizá sea pertinente flexibilizar algunos de esos principios para poder así abrir su campo de acción. De este modo podrían incluirse dentro de la categoría "agroecológica" aquellas nuevas prácticas agrícolas que, aun no siendo las tradicionales del campesinado, contribuyen de manera efectiva a la sostenibilidad ecológica, social y económica de las áreas rurales, y que, por eso, deberían ser objeto de protección y apoyo.

\section{Bibliografía citada}

Altieri, M.A. (1995). Agroecology: the science of sustainable agriculture. Westview Press, Boulder, USA.

Anderson, C.R.; Pimbert, M.P.; Chappell, M.J.; Brem-Wilson, J.; Claeys, P.; Kiss, C.; Maughan, C.; Milgroom, J.; McAllister, G.; Moeller, N. y Singh, J. (2020). "Agroecology now - connecting the dots to enable agroecology transformations", Agroecology and Sustainable Food Systems, 44:5, 561-565.

Calle, A. y Álvarez, I. (2021). "La agroecología, en marcha”, en XXIX Informe Socioeconómico de la Agricultura Familiar, Fundación de Estudios Rurales, Madrid.

Elzen, B.; Augustyn, A.M.; Barbier, M. y van Mierlo, B. (2017). Agroecological transitions: changes and breakthroughs in the making. Wageningen University \& Research.

FAO (2018). The 10 elements of agroecology: guiding the transition to sustainable food and agricultural systems. http://www.fao.org/3/i9037en/i9037en.pdf

Gallardo-López, F.; Hernández-Chontal, M.A.; Cisneros-Saguilán, P. y Linares-Gabriel, A. (2018). "Development of the concept of agroecology in Europe: a review". Sustainability, 10 (4), 1210.

Gliessman, S. (2018). "Defining agroecology”. Agroecology Sustainable Food Systems, 42:599-600

IPES-Food (2016). From university to diversity. A paradigm shift from industrial agriculture to diversified agroecological systems. International Panel of Experts on Sustainable Food Systems. FAO, Roma. http://www.ipes-food.org/images/Reports/UniformityToDiversity_FullReport.pdf

Lozano, C. y Gómez Benito, C. (2014). “Consumidores o ciudadanos? Reflexiones sobre el concepto de ciudadanía alimentaria”, Panorama social, no 19, pp. 77-89

Moyano Estrada, E. (2021). "Por qué decimos agricultura ecológica cuando queremos decir agroecología?", The Conversation, 18 de enero. https://theconversation.com/por-que-decimos-agricultura-ecologica-cuandoqueremos-decir-agroecologia-150303

Nicholls, C.I.; Altieri, M.A. y Vázquez, L. (2016). “Agroecology: principles for the conversion and redesign of farming systems". Journal of Ecosystems and Ecography S, 5.

Sevilla Guzmán, E. (2021). "La agroecología en perspectiva”, XXIX Informe Socioeconómico de la Agricultura Familiar en España, Fundación de Estudios Rurales, Madrid.

Sinclair, F.; Wezel, A.; Mbow, C.; Chomba, S.; Robiglio, V. y Harrison, R. (2019). The contribution of agroecological approaches to realizing climate-resilient agriculture. GCA: Rotterdam, The Netherlands.

Tornaghi, C. y Dehaene, M. (2020). "The prefigurative power of urban political agroecology: rethinking the urbanisms of agroecological transitions for food system transformation”. Agroecology and Sustainable Food Systems, 44(5), 594-610.

Wezel, A.; Bellon, S.; Doré, T.; Francis, C.; Vallod, D. y David, C. (2009). “Agroecology as a science, a movement and a practice. A review”. Agronomy for sustainable development, 29(4), 503-515.

Wezel, A.; Herren, B.G.; Kerr, R.B.; Barrios, E.; Rodrigues-Gonçalves, A.L., y Sinclair, F. (2020). “Agroecological principles and elements and their implications for transitioning to sustainable food systems. A review". Agronomy for Sustainable Development. 40, 40. 